\title{
Global analysis of H3K27me3 as an epigenetic marker in prostate cancer progression
}

\author{
Marjolaine Ngollo ${ }^{1,2}$, Andre Lebert ${ }^{3}$, Marine Daures ${ }^{1,2}$, Gaelle Judes ${ }^{1,2}$, Khaldoun Rifai $^{1,2}$, Lucas Dubois ${ }^{1,2}$, \\ Jean-Louis Kemeny ${ }^{4}$, Frederique Penault-Llorca ${ }^{2,5}$, Yves-Jean Bignon ${ }^{1,2}$, Laurent Guy ${ }^{2,6}$ \\ and Dominique Bernard-Gallon ${ }^{1,2^{*}}$
}

\begin{abstract}
Background: H3K27me3 histone marks shape the inhibition of gene transcription. In prostate cancer, the deregulation of H3K27me3 marks might play a role in prostate tumor progression.

Methods: We investigated genome-wide H3K27me3 histone methylation profile using chromatin immunoprecipitation (ChIP) and 2X400K promoter microarrays to identify differentially-enriched regions in biopsy samples from prostate cancer patients. H3K27me3 marks were assessed in 34 prostate tumors: 11 with Gleason score $>7$ (GS > 7), 10 with Gleason score $\leq 7$ (GS $\leq 7$ ), and 13 morphologically normal prostate samples.

Results: Here, H3K27me3 profiling identified an average of 386 enriched-genes on promoter regions in healthy control group versus 545 genes in GS $\leq 7$ and 748 genes in GS $>7$ group. We then ran a factorial discriminant analysis (FDA) and compared the enriched genes in prostate-tumor biopsies and normal biopsies using ANOVA to identify significantly differentially-enriched genes. The analysis identified ALG5, EXOSC8, CBX1, GRID2, GRIN3B, ING3, MYO1D, NPHP3-AS1, MSH6, FBXO11, SND1, SPATS2, TENM4 and TRA2A genes. These genes are possibly associated with prostate cancer. Notably, the H3K27me3 histone mark emerged as a novel regulatory mechanism in poor-prognosis prostate cancer.
\end{abstract}

Conclusions: Our findings point to epigenetic mark H3K27me3 as an important event in prostate carcinogenesis and progression. The results reported here provide new molecular insights into the pathogenesis of prostate cancer.

Keywords: ChIP-on-chip, Epigenetics, Histone methylation, H3K27me3, Prostate, Cancer

\section{Background}

Epigenetic alterations play a critical role in cancer initiation and progression in addition to genetic alterations. Epigenetics includes changes such as DNA methylation, microRNA and histone modifications, which together make up the epigenome [1]. Here we focused on the histone modifications that play a crucial role in cancer development and progression. Chromatin structure plays an important role in regulating various nuclear functions such as transcription, replication, recombination and DNA repair. Regulation of gene

\footnotetext{
* Correspondence: dominique.gallon-bernard@clermont.unicancer.fr

'Department of Oncogenetics, Centre Jean Perrin - CBRV, 28 place Henri

Dunant, BP 38, 63001 Clermont-Ferrand, France

${ }^{2}$ INSERM U 1240, IMOST, 58 rue Montalembert-BP184, 63005

Clermont-Ferrand, France

Full list of author information is available at the end of the article
}

expression is known to be involved in the binding of transcription factors in target gene promoters but it is also dependent on how the epigenetic events, including histone marks, are characterized. Basically, the local modification of chromatin structure by histone modifications can lead to activation or inactivation of gene expression. For example, some histone modifications such as tri-methylated histone $\mathrm{H} 3$ at lysine 27 (H3K27me3) are known to inactive gene expression.

Studies of alteration in histone methylation at wholegenome scale bring insight into gene regulation. Global changes in histone $\mathrm{H} 3$ are emerging as a new biomarker in malignant transformation [2, 3]. Likewise, histonemodifier enzymes control dynamic transcription of gene expression in normal and cancer cells, enabling key physiopathological processes to take place $[4,5]$. 
Polycomb-group proteins (PcGs) are involved in silencing gene expression, particularly during development and differentiation stages $[6,7]$, and also play the major role in nuclear reprogramming and chromatin remodeling [8]. PcGs are organized into two main polycomb-repressive complexes (PRCs), PRC1 and PRC2, that control gene silencing through post-translational histone modifications $[9,10]$.

At specific loci, the histone methyltransferase enhancer of zeste homolog 2 (EZH2), a subunit of PRC2, catalyzes H3K27 trimethylation, leading to chromatin compaction and subsequently silencing of genes in prostate cancer [11]. Abnormal functions of PcGs are one of the main factors involved in the initiation and progression phases in many cancers, including prostate cancer [12]. EZH2 is highly overexpressed in prostate cancer and strongly associated with epigenetic silencing in cancer. EZH2 is so prominently involved in aggressive cell growth, metastasis, drug resistance and stem cell maintenance that it has become an attractive therapeutic target in prostate cancer [13-15]. Previous studies show that EZH2 up-regulation is correlated with H3K27me3 deregulation and poor-prognosis prostate tumor [16]. The H3K27me3 repressive mark has been found on many gene promoters that are silenced [17], and genome-wide profiling studies of the H3K27me3 mark in metastatic and prostate cancer cells suggest a silencing function of EZH2 in prostate cancer [18, 19].

This study used 34 human prostate biopsies and chromatin immunoprecipitation (ChIP) assays to investigate the interactions between $\mathrm{H} 3 \mathrm{~K} 27 \mathrm{me} 3$ and gene promoter in prostate cancer. ChIP coupled with promoter microarrays enabled us to determine the entire spectrum of in vivo DNA binding sites of the H3K27me3 repressive mark. Identifying region-specific H3K27me3 patterns also helps address additional questions, such as how these observations may resolve or at least suggest causal relationships between histone methylation and prostate cancer progression.

We demonstrated that average number of H3K27me3enriched genes was higher in tumor tissues than normal tissues. Then, after factorial discriminant analysis and ANOVA, we characterized the significant interaction of H3K27me3 with ALG5, EXOSC8, CBX1, GRID2, GRIN3B, ING3, MYO1D, NPHP3-AS1, MSH6, FBXO11, SND1, SPATS2, TENM4 and TRA2A in tumor tissues compared to normal tissues. These genes were all more H3K27me3-enriched and were able to discriminate different groups according to Gleason score.

\section{Methods}

\section{Prostate patient samples}

Normal and tumoral prostate biopsies were obtained from 34 patients (Table 1) diagnosed with prostate
Table 1 Clinical and biological characteristics of patients

\begin{tabular}{lll}
\hline Cases & $T$ & $\mathrm{NT}$ \\
\hline Total cases $(n=34)$ & 21 & 13 \\
Age at diagnosis (years) & & \\
$\quad<49$ & 0 & 0 \\
$50-59$ & 2 & 2 \\
$60-69$ & 11 & 8 \\
$\quad>70$ & 8 & 3 \\
Baseline PSA (ng/mL) & & \\
$<4$ & 0 & 0 \\
$4-10$ & 8 & 4 \\
$10-20$ & 8 & 0 \\
$>20$ & 5 & - \\
Clinical stage & & - \\
T1c & 7 & - \\
T2 & 8 & - \\
T3 & 6 & - \\
Gleason Score & & \\
$\leq 7$ & 10 & \\
$>7$ & 11 & \\
\hline
\end{tabular}

$\pi$ tumoral tissues, NT normal tissues, PSA prostate-specific antigen

cancer at Clermont-Ferrand University Hospital (France). All biopsies were kept in nitrogen. A pathologist performed tumor evaluation. Patients did not receive chemotherapy before clinical examination. All subjects gave written informed consent to the study, which was approved by the French Ministry for Higher Education and Research (DC-2008-558).

\section{Chromatin immunoprecipitation (ChIP)}

Tissues were fixed for $15 \mathrm{~min}$ at room temperature (RT) using $1 \%$ formaldehyde in phosphate buffered saline (PBS) containing protease inhibitors. Reversal of crosslinking was performed by incubation with $0.125 \mathrm{M}$ glycine for $5 \mathrm{~min}$ at RT. Each pellet was re-suspended with lysis buffer (5 mM PIPES pH 8, $0.85 \mathrm{mM}$ KCL, $0.5 \%$ Igepal) supplemented with $1 \mathrm{X}$ protease inhibitor cocktail, and sonicated for $30 \mathrm{~min}$ in $30 \mathrm{~s}$ ON/30 s OFF cycles (Bioruptor, Diagenode). The lysate was centrifuged at $14,000 \mathrm{~g}$ for $10 \mathrm{~min}$ and the supernatant transferred to a fresh tube. Optimal fragmentation was achieved by testing various sonication conditions on chromatin followed by DNA isolation and gel electrophoresis estimation of sonication efficiency. ChIP was performed using an AutoTrue MicroChIP kit (Diagenode \#C01010140) on a SX-8G IP-Star Compact Automated System (Diagenode) as per the manufacturer's instructions. Immunoprecipitation was performed using $3 \mu \mathrm{g}$ of anti-H3K27me3 (Diagenode \#C15410195) and nonspecific IgG (Diagenode). Reverse crosslinking was 
performed with $5 \mathrm{M} \mathrm{NaCl}$ for $4 \mathrm{~h}$ at $65{ }^{\circ} \mathrm{C}$. The immunoprecipitated DNA and input samples were purified using MicroChIP DiaPure columns (Diagenode \#C03040001) and eluted with TE buffer. After ChIP, the crosslink was reversed and the DNAs were purified. To assess the quality and efficiency of the ChIP procedure, quantitative PCR was performed to assess the enrichment of known target genes. GAPDH, a housekeeping gene, was used as negative control for H3K27me3 ChIP. TSH2B gene, which is present in heterochromatin, was used as positive control for H3K27me3. TSH2B showed strong enrichment of H3K27me3 while GAPDH gene showed weak enrichment. Only samples with an enrichment of H3K27me3 above 5 were selected for ChIP-on-Chip analyses. Quantitative PCR was performed using SYBR Green Mix (Applied Biosystems \#4309155) following the manufacturer's instructions. The samples were amplified using an Applied Biosystems ABI Prism ${ }^{\circ} 7900$ HT Real-Time PCR System (Applied Biosystems). PCR program was $95{ }^{\circ} \mathrm{C}$ for $3 \mathrm{~min}$ and 40 cycles of $95{ }^{\circ} \mathrm{C}$ for $30 \mathrm{~s}, 60{ }^{\circ} \mathrm{C}$ for $30 \mathrm{~s}$ and $72{ }^{\circ} \mathrm{C}$ for $30 \mathrm{~s}$. The IP and input DNA were then subjected to microarray hybridization.

\section{Promoter microarray hybridization}

After ChIP, the immunoprecipitated DNA was preamplified with a whole-genome amplification kit (Sigma \#WGA2) following the manufacturer's protocol, and $2 \mu \mathrm{g}$ DNA was labeled using a SureTag complete DNA labeling kit (Agilent Technologies \#51904240) at $37{ }^{\circ} \mathrm{C}$ for $2 \mathrm{~h}$ then $65{ }^{\circ} \mathrm{C}$ for $10 \mathrm{~min}$. Input DNA was then labeled with cyanine 3 while immunoprecipitated DNA was labeled with cyanine 5 . Both samples were purified on columns and eluted in TE buffer. Labeled DNAs were mixed and competitively hybridized to DNA microarrays. Hybridization was carried out on 2X400K Sure Print G3 Human promoter microarrays (Agilent \#G4874A) in the presence of human Cot-1 DNA for $40 \mathrm{~h}$ at $65^{\circ} \mathrm{C}$ and the slides were washed according to Agilent's procedure. After washing, the slides were scanned using an Agilent microarray scanner, and intensity of fluorescent signals was extracted using Agilent feature extraction 11.2 software.

Each slide contained two identical arrays and each microarray contained 414,043 (60-mer) oligonucleotide probes spaced every 172 bp across promoter regions including $-5.5 \mathrm{~Kb}$ upstream and $+2.5 \mathrm{~Kb}$ downstream of identified transcriptional start sites (TSS). The probes covered 21,000 of the best-defined human transcripts represented as RefSeq genes.

\section{Data analysis}

For the H3K27me3-enriched gene analysis between normal tissues and tumors, ChIP-on-chip data were processed using RINGO software 1.26.1, then the microarray data was analyzed on $\mathrm{R}$ software using several Bioconductor packages (www.bioconductor.org/). Enriched regions were defined from enriched probes using the criteria of at least 3 enriched probes within the region. For each probe on the array, a score was calculated as follows: score $=\sum$ (enrichment values Probes-Enrichment Threshold). Only genes with a threshold of $>1.5$ were considered as differently enriched. Genes with a score of least than 1.5 were removed from analysis, as were genes with missing data in more than $30 \%$ of the samples.

\section{Gene annotation}

Gene annotation was carried out using the ENSEMBL annotation system. We generated enrichment profiles for H3K27me3 in tumor samples compared to normal tissues. After determining the enriched regions for H3K27me3 modifications, RefSeq genes were downloaded from the ENSEMBL database.

\section{Statistical analysis}

H3K27me3 sites were defined as differentially enriched if the Enrichment Score was $>1.5$, and for each H3K27me3 site the mean Enrichment Score level was compared in tumor tissue group versus normal tissue. Factorial discriminant analysis (FDA) and ANOVA were performed to discriminate the three groups. Data was analyzed using $\mathrm{R}$ statistics. Thresholds set for statistical significance were $* p<0.05$ and ${ }^{* *} p<0.01$.

\section{Results}

Whole-genome screening of H3K27me3 epigenetic marks in human prostate cancer

In order to grasp the role H3K27me3 marks in prostate cancer progression in 34 patients, we investigated H3K27me3 mark binding to determine whether it correlates with tumor progression. First, to examine the epigenetic signature of H3K27me3 in prostate cancer, we mapped the global promoter occupancy profile of H3K27me3 in prostate cancer compared to normal biopsies using ChIP-on-chip methods.

Samples were divided into three groups: 13 normal prostate tissues, 11 prostate cancer tissues with Gleason score $>7$ and 10 prostate cancer tissues with Gleason score $\leq 7$. Enriched regions were defined via an Enrichment Score (ES)-based sliding window approach using RINGO software [20], then the binding sites were annotated to human genes using the ENSEMBL database. Importantly, we selected genes whose enrichment score was greater than 1.5 in the promoter regions. Among the 21,000 genes analyzed in human 2X400K, we calculated the average of H3K27me3 modifications among patients in each group. We identified an average of 386 genes with H3K27me3 marks in the promoter regions in healthy control group versus 545 genes in 
GS $\leq 7$ and 748 genes in GS $>7$ group. These results suggested that there are more extensive H3K27me3enriched gene promoters in advanced disease than normal tissues.

To list enriched-genes, we performed a hierarchic clustering analysis that also helped to see similarities between patients. The genome-scale H3K27me3 profile of each group was then compared. Figure 1 showed the enrichment of genes such as IFIH1, RCN1, XRN2, EIF2B, RP11-156P1.3 and AC079305.11. However, differences at the genetic and molecular level could explain by the interindividual difference in control group. Interestingly, all of these genes are shared with all patients in GS $\leq 7$ group. Despite interindividual variability, we identified one gene that is specific to GS $\leq 7$ group, TRA2A gene (Fig. 2).

The greatest changes occurred in GS $>7$ group where we observed several H3K27me3-enriched genes such as MGMT, SLC4A4, ABHD2, PAPOLG, NSF, ING3, TMPRSS6, FNDC3B (Fig. 3).

\section{H3K27me3 epigenetic marks correlate with prostate cancer aggressiveness}

Discriminant analysis on the whole microarray dataset showed a clear segregation of samples with GS $\leq 7$, GS $>7$ and healthy controls. This analysis indicated that H3K27me3 profiles could classify prostate cancer patients (Fig. 4).

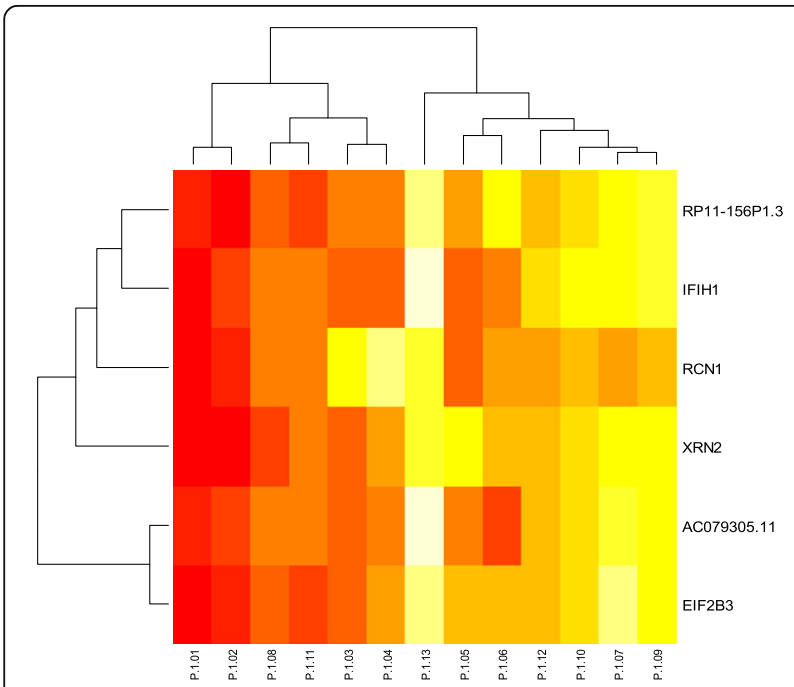

Fig. 1 Hierarchical clustering on a set of 13 normal biopsies. The scaled enrichment score of individual patients is plotted in a red-yellow scale. Color intensity reflects magnitude of enrichment score, with red indicating high H3K27me3 enrichment and yellow indicating low H3K27me3 enrichment. Columns represent individual tissues. Rows represent the genes. The dendogram represents overall similarities in patient profiles

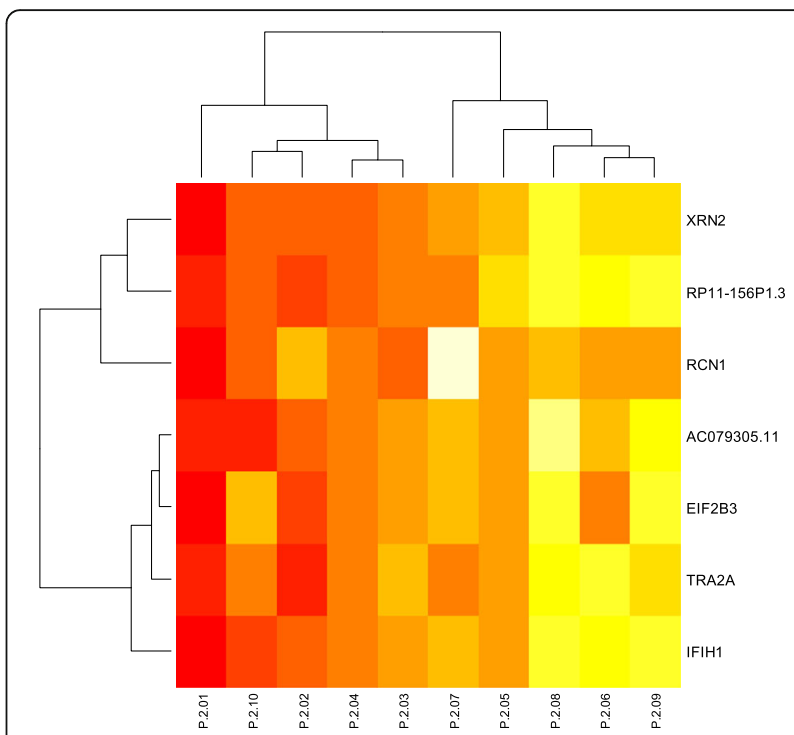

Fig. 2 Hierarchical clustering analysis of tumor tissues with Gleason score $\leq 7$. The scaled enrichment score of individual patients is plotted in a red-yellow scale. Color intensity reflects magnitude of enrichment score, with red indicating high H3K27me3 enrichment and yellow indicating low H3K27me3 enrichment. Columns represent individual tissues and rows represent the genes. The dendogram represents overall similarities in patient profiles

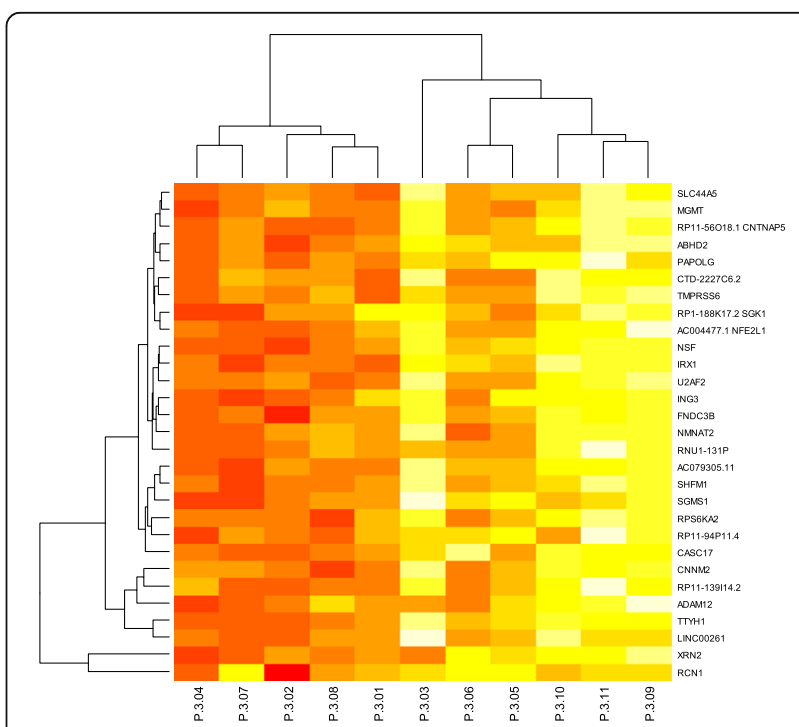

Fig. 3 Hierarchical clustering analyses of patients with Gleason score $>7$. The scaled enrichment score of individual patients is plotted in a red-yellow scale. Color intensity reflects magnitude of enrichment score, with red indicating high H3K27me3 enrichment and yellow indicating low H3K27me3 enrichment. Columns represent individual tissues. Rows represent the genes. The dendogram represents overall similarities in patient profiles 


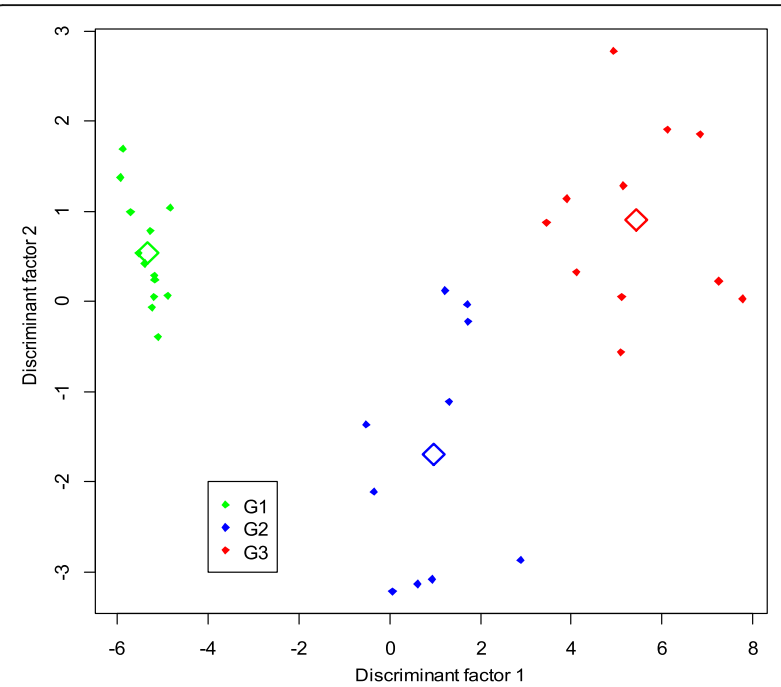

Fig. 4 Factorial discriminant analysis (FDA) of microarray-based genome-wide H3K27me3 profiles derived from prostate biopsies. Prostate biopsies were obtained from healthy patients $(n=13)$, prostate cancer patients with Gleason score $\leq 7(n=10)$ and prostate cancer patients with Gleason score $>7(n=11)$. Data showed a well-defined separation between patients according to Gleason score and H3K27me3 markers. Center of gravity for each group is reported as the empty symbol. G1, healthy group; G2, prostate cancer with Gleason score $\leq 7 ; \mathrm{G} 3$ prostate cancer with Gleason score $>7$

Enriched genes with H3K27me3 marks in prostate cancer tissues versus normal tissues

Even though the global pattern of enriched genes in carcinoma tissues and normal tissues showed variability between patients, it was possible to identify differentially H3K27me3-enriched genes involved in prostate cancer based on enrichment score level. Using ANOVA, we identified genes that were significantly enriched between both tumor groups versus normal samples. The significance of the enrichment score values of $A L G 5$, EXOSC8, CBX1, GRID2, GRIN3B, ING3, MYO1D, NPHP3-AS1, MSH6, FBXO11, SND1, SPATS2, TENM4 and TRA2A genes are shown in Table 2. Association of H3K27me3 enrichment and clinico-pathological variables like stage and PSA level did not show any significance. However, only Gleason score correlated with the H3K27me3 enrichment on genes (Fig. 5).

\section{Discussion}

There is a pressing need for further work on the molecular mechanisms underlying prostate cancer in order to improve prognosis, diagnosis and treatment. In particular, characterizing the functional role of genetics in prostate cancer by observing the new target gene would help identify potential drugs. Here we report a set of target genes that interact with $\mathrm{H} 3 \mathrm{~K} 27 \mathrm{me} 3$ in prostate cancer.
Table 2 Compiled statistics of FDA and ANOVA results

\begin{tabular}{lllll}
\hline Gene name & $\begin{array}{l}\text { Coordinate } \\
\text { axis } 1\end{array}$ & $\begin{array}{l}\text { Coordinate } \\
\text { axis } 2\end{array}$ & $p$ value & Significance \\
\hline ALG5/EXOSC8 & 0.815 & -0.154 & 0.001 & $* *$ \\
CBX1 & 0.643 & 0.079 & 0.038 & $*$ \\
GRID2 & 0.655 & 0.056 & 0.034 & $*$ \\
GRIN3B & 0.627 & -0.168 & 0.039 & $*$ \\
ING3 & 0.074 & 0.748 & 0.020 & $*$ \\
MYO1D & 0.666 & -0.168 & 0.023 & $*$ \\
NPHP3-AS1 & 0.640 & -0.186 & 0.031 & $*$ \\
MSH6/FBXO11 & 0.366 & 0.561 & 0.047 & $*$ \\
SND1 & 0.187 & 0.653 & 0.049 & $*$ \\
SPATS2 & 0.579 & -0.352 & 0.031 & $*$ \\
TENM4 & 0.592 & 0.320 & 0.032 & $*$ \\
TRA2A & 0.563 & -0.412 & 0.025 & $*$
\end{tabular}

Coordinate axes refer to FDA data values (Fig. 4). $P$ value refers to ANOVA data. ${ }^{*}<0.05{ }^{* *}<0.01$

Differentially H3K27me3-enriched genes in prostate cancer tissues compared to normal biopsies

Comparison of the H3K27me3 profiles of prostate cancer tissues versus normal tissues revealed an average of 386 enriched-genes on promoter regions in healthy control group versus 545 genes in GS $\leq 7$ and 748 genes in GS > 7 group. These data characterize H3K27me3 as an epigenetic feature of histone methylation-related prostate cancer progression. For the study design and criteria used here, patients were pooled at every stage analyzed to reduce the number of non-common genes. This pooling brought together individuals showing the lowest disparity of results in each group. The goal was

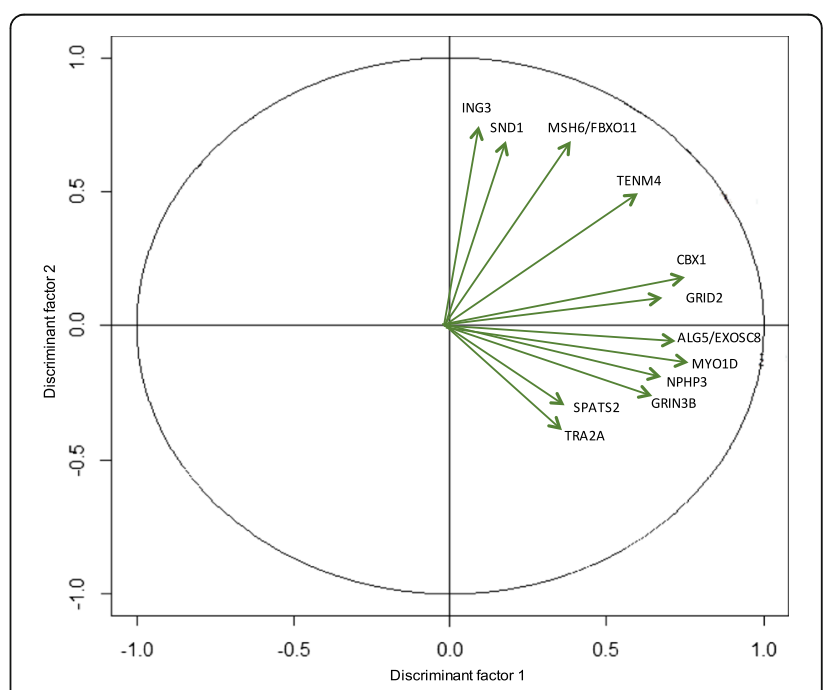

Fig. 5 Factorial discriminant analysis. The results represent differentially H3K27me3-enriched genes between prostate cancer tissues versus normal tissues. The Highly enrichment correlated with GS > 7 
to identify common genes that may be significant and representative of any disease stage.

Among the genes identified as being differentially regulated by H3K27me3 were TRA2A, FBXO11, ING3 and CBX1.

Note that TRA2A gene was shown to discriminate control group and GS $\leq 7$ group. TRA2A plays a role in the regulation of pre-mRNA splicing after phosphorylation and binding to specific RNA [21, 22]. This gene has not been studied yet in connection with prostate tumorigenesis. Furthermore, TRA2A appeared to be H3K27me3-enriched in all patients and could thus serve as an epigenetic marker for early prostate cancer screening. In contrast, GS > 7 patients showed high H3K27me3 enrichment at the TRA2A promoter compared to both the GS $\leq 7$ and normal groups, suggesting its major role in prostate cancer.

FBXO11, ING3 and CBX1 genes all play a role in epigenetic control and regulation of chromatin. FOBXO11 is an arginine methyltransferase that symmetrically dimethylates arginine residues. A recent study in epithelial cancer demonstrated cells that FBXO11 induced an increase of Snail and a decrease of E-cadherin to prevent tumor progression, thus characterizing FBXO11 as a tumor suppressor [23]. H3K27me3 enrichment on the $F B X O 11$ promoter may mediate the repression of this gene in prostate cancer.

The other tumor suppressor gene characterized here was ING3. This inhibitor of growth-family protein was initially identified as a tumor suppressor with altered regulation in a variety of cancer types, including in colorectal cancer cells $[24,25]$. ING is, however, a protein involved in chromatin remodeling. In fact, ING3 acts as a reader of epigenetic code through specific recognition of H3K4me3 and can affect HAT and HDAC activity by serving as members of the Sin3A, Tip60 or Moz/Morf HAT complexes [26]. Our results showed the epigenetic regulation of ING3 via H3K27me3 in prostate cancer suggesting putative tumor suppressor gene silencing by histone methylation in prostate cancer. These data suggest that the ING3 locus may locate in bivalent domains marked by both H3K27me3 and H3K4me3 and that ING3 may thus play a critical role in cancer development.

CBX1 ( $H P 1 \alpha)$ is a member of the heterochromatin protein 1 family (HP1s) that plays a role in the formation and maintenance of heterochromatin. This gene encodes a non-histone protein that is able to bind to histone proteins via methylated lysine residues. Genome-wide localization analysis reveals H3K27me3 binding at CBX1 promoter regions and thus points to heterochromatin formation corresponding to gene silencing in prostate cancer. It has already been shown that CBX1 is downregulated in invasive breast cancer cells [27], and our findings show that a novel epigenetic mechanism might involve CBX1 in transcriptional regulation, thus providing new insight for further elucidation of the molecular mechanisms causing the $C B X 1$ downregulation in cancer cells.

Other genes found to be H3K27me3-enriched in prostate cancer tissues compared to normal tissues include MYO1D, TENM4, GRIN3B, all of which are involved in cell communication and cell adhesion [28-30]. These genes have not yet been described in human cancer, but disrupted intracellular adhesion is a prerequisite for tumor cell invasion and metastasis.

Furthermore, MSH6 gene was found to be epigenetically regulated in prostate cancer. MSH6 is DNA mismatch repair genes. The loss-of-function DNA mismatch repair genes are linked to mutation or epigenetic silencing [31]. In addition, the hypermutated subtype of prostate cancer is chiefly due to loss-offunction mutations in MSH6 in advanced prostate cancer [32]. We thus hypothesized that transcriptionallyrepressed MSH6 gene might be related to H3K27me3 epigenetic modification in prostate cancer.

The comparison of normal and tumor prostate samples revealed far more H3K27me3 marks in advanced tumor tissues compared to normal tissues. These alterations could have major impacts on global gene expression via chromatin state. Our observations suggested that H3K27me3 marks are active in tumor tissues. Increased H3K27me3 marks could be explained by the activity of the PcG such as EZH2, which is frequently over-expressed in prostate cancer [13, 33]. These results implied that the most numerous epigenetic changes from normal tissues to prostate cancer tissues were gains of H3K27me3 marks.

Although the results reported here cannot confirm a repressor status on the increase of H3K27me3 marks on genes, they can serve to formulate a hypothesis. Performing qPCR to validate the selected differentially-enriched genes would help gauge the reliability of the ChIP-on-chip data reported here. Chromatin accessibility could be analyzed by ChIP-qPCR with RNA polymerase II. A previous study had shown that combinations of histone marks, for example gain of H3K4me3 and loss of H3K27me3 or gain of H3K27me3 and loss of H3K4me3, were strongly associated with up-regulated and down-regulated genes in prostate cancer cells. Nevertheless, gain or loss of just one mark is unlikely to prove sufficient for transcriptional changes [34].

\section{Conclusions}

The findings of this study provide key insight for elucidating the regulation of epigenetic changes in prostate cancer. We demonstrated that global H3K27me3 histone modifications correlated with Gleason score in prostate cancer. A set of epigenetic markers was identified, and the data suggests a complex interplay between EZH2 and H3K27me3 histone modifications. 


\section{Abbreviations}

ChIP: Chromatin immunoprecipitation; EZH2: Enhancer of zeste homolog 2; FDA: Factorial discriminant analysis; GS: Gleason score; H3K27me3: Histone 3 lysine 27 trimethylation; H3K4me3: Histone 4 lysine 4 trimethylation; HAT: Histone acetyltransferase; HDAC: Histone deacetylase; HP1: Heterochromatin protein 1 family; PBS: Phosphate buffered saline; PCGs: Polycomb-group proteins; PRC: Polycomb-repressive complex; TSS: Transcriptional start sites

\section{Acknowledgements}

Biological biopsies were centralized in Biological Resource Center of Centre Jean Perrin accredited under No. BB-0033-00075. We thank la Ligue contre le Cancer, comités du Puy de Dôme et de la Haute-Loire.

\section{Funding}

Not applicable.

\section{Availability of data and materials}

The cohort data are available to researchers. The other datasets supporting the conclusions of this article are included within the article.

\section{Authors' contributions}

$B G D, G L, B Y J, P L F$ conceived and designed the research. NM, JG, DM, RK, DL performed the experiments. NM, LA, GL, BGD analyzed the data. LA performed the statistical analysis. NM and BGD wrote the paper. KJL and PLF carried out anatomopathological examinations. All authors read and approved the final manuscript.

\section{Competing interests}

The authors declare that they have no competing interests.

\section{Consent for publication}

Not applicable.

\section{Ethics approval and consent to participate}

The samples were obtained from Clermont-Ferrand University Hospital (France) and a prior signed informed consent was obtained from each patient. It was approved by the French Ministry for Higher Education and Research (DC-2008-558).

\section{Publisher's note}

Springer Nature remains neutral with regard to jurisdictional claims in published maps and institutional affiliations.

\section{Author details}

Department of Oncogenetics, Centre Jean Perrin - CBRV, 28 place Henri Dunant, BP 38, 63001 Clermont-Ferrand, France. ${ }^{2}$ INSERM U 1240, IMOST, 58 rue Montalembert-BP184, 63005 Clermont-Ferrand, France. ${ }^{3}$ University Blaise Pascal, Institut Pascal UMR 6602 CNRS/UBP, 24 Avenue des Landais, Aubière, France. ${ }^{4}$ Department of Biopathology, Gabriel Montpied Hospital, 58 rue Montalembert, 63000 Clermont-Ferrand, France. ${ }^{5}$ Department of Biopathology, Centre Jean Perrin, 58 rue Montalembert, 63000 Clermont-Ferrand, France. ${ }^{6}$ Department of Urology, Gabriel Montpied Hospital, 58 rue Montalembert, 63000 Clermont-Ferrand, France.

Received: 20 September 2016 Accepted: 1 April 2017

Published online: 12 April 2017

\section{References}

1. Albany C, Alva AS, Aparicio AM, Singal R, Yellapragada S, Sonpavde G, Hahn NM. Epigenetics in prostate cancer. Prostate Cancer. 2011;2011:580318.

2. Dagdemir A, Durif J, Ngollo M, Bignon YJ, Bernard-Gallon D. Histone lysine trimethylation or acetylation can be modulated by phytoestrogen, estrogen or anti-HDAC in breast cancer cell lines. Epigenomics. 2013;5(1):51-63.

3. Ngollo M, Dagdemir A, Judes G, Kemeny JL, Penault-Llorca F, Boiteux JP, Lebert A, Bignon YJ, Guy L, Bernard-Gallon D. Epigenetics of prostate cancer: distribution of histone H3K27me3 biomarkers in peri-tumoral tissue. OMICS. 2014;18(3):207-9.

4. Guerra-Calderas L, Gonzalez-Barrios R, Herrera LA, Cantu de Leon D, SotoReyes $\mathrm{E}$. The role of the histone demethylase KDM4A in cancer. Cancer Genet. 2015;208(5):215-24
5. Chen Z, Wang L, Wang Q, Li W. Histone modifications and chromatin organization in prostate cancer. Epigenomics. 2010:2(4):551-60.

6. Khan AA, Lee AJ, Roh TY. Polycomb group protein-mediated histone modifications during cell differentiation. Epigenomics. 2015;7(1):75-84

7. Sauvageau M, Sauvageau G. Polycomb group proteins: multi-faceted regulators of somatic stem cells and cancer. Cell Stem Cell. 2010;7(3):299-313.

8. Kerppola TK. Polycomb group complexes-many combinations, many functions. Trends Cell Biol. 2009;19(12):692-704.

9. Lanzuolo C, Orlando V. Memories from the polycomb group proteins. Annu Rev Genet. 2012;46:561-89.

10. Cao R, Wang L, Wang $H$, Xia L, Erdjument-Bromage $H$, Tempst $P$, Jones RS, Zhang Y. Role of histone H3 lysine 27 methylation in Polycomb-group silencing. Science. 2002;298(5595):1039-43.

11. Margueron R, Reinberg D. The Polycomb complex PRC2 and its mark in life. Nature. 2011:469(7330):343-9.

12. Ren G, Baritaki S, Marathe H, Feng J, Park S, Beach S, Bazeley PS, Beshir AB, Fenteany $G$, Mehra $R$, et al. Polycomb protein EZH2 regulates tumor invasion via the transcriptional repression of the metastasis suppressor RKIP in breast and prostate cancer. Cancer Res. 2012;72(12):3091-104.

13. Varambally S, Dhanasekaran SM, Zhou M, Barrette TR, Kumar-Sinha C, Sanda MG, Ghosh D, Pienta KJ, Sewalt RG, Otte AP, et al. The polycomb group protein $\mathrm{EZH} 2$ is involved in progression of prostate cancer. Nature. 2002:419(6907):624-9.

14. Deb G, Thakur VS, Gupta S. Multifaceted role of EZH2 in breast and prostate tumorigenesis: epigenetics and beyond. Epigenetics. 2013:8(5):464-76.

15. Yang YA, Yu J. EZH2, an epigenetic driver of prostate cancer. Protein Cell. 2013:4(5):331-41.

16. Ezponda T, Licht JD. Molecular pathways: deregulation of histone h3 lysine 27 methylation in cancer-different paths, same destination. Clin Cancer Res. 2014:20(19):5001-8

17. Ngollo M, Lebert A, Dagdemir A, Judes G, Karsli-Ceppioglu S, Daures M, Kemeny JL, Penault-Llorca F, Boiteux JP, Bignon YJ, et al. The association between histone 3 lysine 27 trimethylation (H3K27me3) and prostate cancer: relationship with clinicopathological parameters. BMC Cancer. 2014;14:994

18. Kondo Y. Epigenetic cross-talk between DNA methylation and histone modifications in human cancers. Yonsei Med J. 2009;50(4):455-63.

19. Yu J, Rhodes DR, Tomlins SA, Cao X, Chen G, Mehra R, Wang X, Ghosh D, Shah $\mathrm{RB}$, Varambally $\mathrm{S}$, et al. A polycomb repression signature in metastatic prostate cancer predicts cancer outcome. Cancer Res. 2007:67(22):10657-63.

20. Toedling J, Skylar O, Krueger T, Fischer JJ, Sperling S, Huber W. Ringo-an R/ Bioconductor package for analyzing ChIP-chip readouts. BMC Bioinformatics. 2007;8:221.

21. Grosso AR, Martins S, Carmo-Fonseca M. The emerging role of splicing factors in cancer. EMBO Rep. 2008;9(11):1087-93.

22. Witkin KL, Hanlon SE, Strasburger JA, Coffin JM, Jaffrey SR, Howcroft TK, Dedon PC, Steitz JA, Daschner PJ, Read-Connole E. RNA editing, epitranscriptomics, and processing in cancer progression. Cancer Biol Ther 2015:16(1):21-7.

23. Jin $Y$, Shenoy AK, Doernberg $S$, Chen $H$, Luo H, Shen $H$, Lin T, Tarrash M, Cai $\mathrm{Q}, \mathrm{Hu} \mathrm{X}$, et al. FBXO11 promotes ubiquitination of the snail family of transcription factors in cancer progression and epidermal development. Cancer Lett. 2015:362(1):70-82.

24. Nabbi A, Almami A, Thakur S, Suzuki K, Boland D, Bismar TA, Riabowol K ING3 protein expression profiling in normal human tissues suggest its role in cellular growth and self-renewal. Eur J Cell Biol. 2015;94(5):214-22.

25. Gou WF, Sun HZ, Zhao S, Niu ZF, Mao XY, Takano Y, Zheng HC Downregulated inhibitor of growth 3 (ING3) expression during colorectal carcinogenesis. Indian J Med Res. 2014;139(4):561-7.

26. Doyon Y, Cayrou C, Ullah M, Landry AJ, Cote V, Selleck W, Lane WS, Tan S, Yang XJ, Cote J. ING tumor suppressor proteins are critical regulators of chromatin acetylation required for genome expression and perpetuation. Mol Cell. 2006;21(1):51-64

27. Thomsen R, Christensen DB, Rosborg S, Linnet TE, Blechingberg J, Nielsen AL. Analysis of HP1alpha regulation in human breast cancer cells. Mol Carcinog. 2011:50(8):601-13.

28. Sood S, Patel FD, Ghosh S, Arora A, Dhaliwal LK, Srinivasan R. Epigenetic alteration by DNA Methylation of ESR1, MYOD1 and hTERT Gene promoters is useful for prediction of response in patients of locally advanced invasive cervical carcinoma treated by Chemoradiation. Clin Oncol (R Coll Radiol). 2015;27(12):720-7. 
29. Hor H, Francescatto L, Bartesaghi L, Ortega-Cubero S, Kousi M, LorenzoBetancor O, Jimenez-Jimenez FJ, Gironell A, Clarimon J, Drechsel O, et al. Missense mutations in TENM4, a regulator of axon guidance and central myelination, cause essential tremor. Hum Mol Genet. 2015;24(20):5677-86.

30. Lin YT, Hsieh MH, Liu CC, Hwang TJ, Chien YL, Hwu HG, Liu CM. A recentlydiscovered NMDA receptor gene, GRIN3B, is associated with duration mismatch negativity. Psychiatry Res. 2014;218(3):356-8.

31. Grindedal EM, Moller P, Eeles R, Stormorken AT, Bowitz-Lothe IM, Landro SM, Clark N, Kvale R, Shanley S, Maehle L. Germ-line mutations in mismatch repair genes associated with prostate cancer. Cancer Epidemiol Biomark Prev. 2009;18(9):2460-7.

32. Pritchard CC, Morrissey C, Kumar A, Zhang X, Smith C, Coleman I, Salipante SJ, Milbank J, Yu M, Grady WM, et al. Complex MSH2 and MSH6 mutations in hypermutated microsatellite unstable advanced prostate cancer. Nat Commun. 2014:5:4988.

33. Kuser-Abali G, Alptekin A, Cinar B. Overexpression of MYC and EZH2 cooperates to epigenetically silence MST1 expression. Epigenetics. 2014;9(4):634-43.

34. Ke XS, Qu Y, Rostad K, Li WC, Lin B, Halvorsen OJ, Haukaas SA, Jonassen I, Petersen K, Goldfinger N, et al. Genome-wide profiling of histone h3 lysine 4 and lysine 27 trimethylation reveals an epigenetic signature in prostate carcinogenesis. PLoS One. 2009;4(3):e4687.

\section{Submit your next manuscript to BioMed Central} and we will help you at every step:

- We accept pre-submission inquiries

- Our selector tool helps you to find the most relevant journal

- We provide round the clock customer support

- Convenient online submission

- Thorough peer review

- Inclusion in PubMed and all major indexing services

- Maximum visibility for your research

Submit your manuscript at www.biomedcentral.com/submit 